\title{
The Effects of Transcranial Direct-Current Stimulation on Cognition in Stroke Patients
}

\author{
Gi Jeong Yun, ${ }^{a}$ Min Ho Chun, ${ }^{a}$ Bo Ryun Kim ${ }^{\mathrm{b}}$ \\ ${ }^{a}$ Department of Rehabilitation Medicine, Asan Medical Center, University of Ulsan College of Medicine, Seoul, Korea \\ bDepartment of Rehabilitation Medicine, Jeju National University Hospital, Jeju, Korea
}

Background and Purpose To investigate whether transcranial direct-current stimulation (tDCS) can improve cognition in stroke patients.

Methods Forty-five stroke patients (20 males and 25 females, average age: 62.7 years) with cognitive dysfunction were included in this prospective, double-blinded, randomized casecontrol study. All patients were right-handed and the mean elapsed time after stroke was 39.3 days. Three different treatments groups were used: (1) anodal stimulation of the left anterior temporal lobe, (2) anodal stimulation of the right anterior temporal lobe, and (3) sham stimulation. tDCS was delivered for 30 minutes at $2 \mathrm{~mA}$ with $25 \mathrm{~cm}^{2}$ electrodes, five times/week, for a total of 3 weeks, using a Phoresor II Auto Model PM 850 (IOMED ${ }^{\circledR}$ ). The evaluation of cognitive impairment was based on a Computerized Neuropsychological Test (CNT), Korean Mini-Mental State Examination (K-MMSE). The Korean version of the Modified Barthel Index (K-MBI) was used to assess activities of daily living functionality. These evaluations were conducted in all patients before and after treatment.

Results Each group included 15 patients. Pre-treatment evaluation showed no significant differences between the three groups for any of the parameters. There was significant improvement in the verbal learning test on the CNT in the left anodal stimulation group $(P<0.05)$. There were, however, no significant differences in the K-MMSE or K-MBI scores among the three groups.

Conclusions These results demonstrated the beneficial effects of anodal tDCS on memory function. Thus, tDCS can successfully be used as a treatment modality for patients with cognitive dysfunction after stroke.

Keywords Cognition; Stroke; Transcranial direct stimulation

\author{
Correspondence: Min Ho Chun \\ Department of Rehabilitation Medicine, \\ Asan Medical Center, University of Ulsan \\ College of Medicine, 88 Olympic-ro \\ 43-gil, Songpa-gu, Seoul 05505, Korea \\ Tel: $+82-2-3010-3800$ \\ Fax: +82-2-3010-6964 \\ E-mail:mhchun@amc.seoul.kr
}

Received: December 18, 2014

Revised: March 12, 2015

Accepted: April 22, 2015

The authors have no financial conflicts of interest.

\section{Introduction}

Treating cognitive impairment, along with motor recovery and improving performance in daily activities, is one of the key focus areas of stroke rehabilitation. As cognition is closely related to patient motivation and motor function improvement, implementing cognitive function treatment in a timely and effective manner is crucial for the overall success of poststroke reha- bilitation. ${ }^{1}$ Previous reports showed $25 \%-40 \%$ of patients suffer from dementia by three months after stroke, and the number increased to $50 \%-75 \%$ when patients with minor symptoms of cognitive impairment are included..$^{2-4}$ Among patients with brain lesions, memory disturbance is commonly a critical challenge in rehabilitating patients to enable them to return to active participation in society and the workforce. Research has suggested that rehabilitation programs designed to maximize neuroplas- 
ticity are effective for improving memory functions in these patients. $^{5}$

Recently, transcranial direct-current stimulation ( $\mathrm{tDCS}$ ) and repetitive transcranial magnetic stimulation (rTMS), noninvasive methods for stimulating the cerebral cortex, have been intensively studied as a treatment for brain injury. While both modalities are used to treat brain injury, $\mathrm{tDCS}$ is the preferred method, as it allows simultaneous application of brain stimulation and conventional rehabilitation. $\mathrm{tDCS}$ is also easier to implement with fewer side effects than rTMS.

Boggio et al. ${ }^{6}$ concluded that applying tDCS to the brain region improved motor functions and increased motor cortex excitability. Recent studies also suggested that $\mathrm{tDCS}$ was useful in treating cognitive dysfunction, and improving attention, memory, and executive function. ${ }^{7-9}$ However, there are still insufficient data to confirm its effect on the cognitive functions of stroke patients. Thus, in this study, we determined the effectiveness of $\mathrm{tDCS}$ in cognitive recovery in acute and subacute stroke patients.

\section{Methods}

\section{Subjects}

We selected stroke patients with cognitive impairment and a score of $\leq 27$ on the Korean version of Mini Mental State Exam (K-MMSE) from patients treated at the Department of Rehabilitation Medicine, Asan Medical Center, from October 1, 2010 to March 30, 2012. The guardians of the patients indicated that the patients had experienced their first stroke, and had no previous history of abnormal cognitive functions. Patients who were classified as acute or subacute, within 6 months from their stroke, and who showed no damage to their temporal lobe on magnetic resonance imaging, were selected. Subjects were then included when they were willing and available for five treatment sessions per week. Patients with apraxia, aphasia, and neglect were excluded, given that these factors may have an impact on the outcome of cognitive function tests. For the safety of patients, we excluded those who had a history of craniectomy or seizure.

\section{Treatments}

We used the double-blind method to randomly divide the subjects into three test groups: (1) the left fronto-temporal anode stimulation (left-FTAS) group, (2) the right fronto-temporal anode stimulation (right-FTAS) group, and (3) the sham group. Patients in each group received tDCS treatment for 30 min, five times a week, for 3 weeks. The treatments were performed using a Phoresor II Auto Model PM850 (IOMED ${ }^{\circledR}$, Salt Lake City, UT, USA), an FDA-approved device, with $5 \times 5 \mathrm{~cm}$ sponge electrodes attached to the patient's scalp. The intensity of the current was set at $2 \mathrm{~mA}$, which was confirmed as safe by Iyer et al., ${ }^{2-4}$ and stimulation was maintained for 30 minutes. tDCS anodal electrodes were placed according to the 10-20 international EEG system, at T3 for the left-FTAS group and at T4 for the right-FTAS group. ${ }^{10,11}$ For the sham group, sponge electrodes were attached using the same method as for the left-FTAS group, but without any current running. The cognitive rehabilitation program used in the study was ComCog (Maxmedica Inc., Seoul, Korea), with the focus on attention and memory improvement for cognitive disorder patients.

The subjects were evaluated before the treatment and after completing the 3-week program, using the K-MMSE and computerized neurocognitive function tests (CNT, MAXMEDICA, Seoul, Korea) to assess cognitive function, and the Korean version of the modified Barthel Index (K-MBI) to assess activities of daily living. The CNT included visual and auditory continuous performance tests (CPTs) to evaluate continuous concentration on visual and auditory stimuli. Forward and backward digit span tests were used to test attention or working memory. Verbal learning tests were used to evaluate verbal memory.

\section{Statistical analysis}

We used SPSS ver. 14.0 for the statistical analysis of data obtained in this study. Descriptive statistics were used to calculate the mean values and standard deviations. The Kruskal-Wallis test was used to test the homogeneity among the three groups, and the Wilcoxon signed-rank test was used to analyze the preand post-treatment data for each test group. The Kruskal-Wallis test was used to compare the three test groups, and the MannWhitney test was used as the follow-up test. The level of significance was set as $P<0.05$.

\section{Results}

\section{General characteristics of the subjects}

The subjects for this study included: (1) six male and nine female patients in the left-FTAS group, (2) seven male and eight female patients in the right-FTAS group, and (3) seven male and eight female patients in the sham group. The mean age of patients in each group was as follows: (1) $60.9 \pm 12.9$ years for the leftFTAS group, (2) $58.9 \pm 15.0$ years for the right-FTAS group, and (3) $68.5 \pm 14.6$ years for the control group, with no significant differences between the groups. The mean time elapsed from the onset of stroke to the start of the treatment program was: (1) $42.2 \pm 31.9$ days for the left-FTAS group, (2) $38.1 \pm 27.0$ days for the right-FTAS group, and (3) $39.5 \pm 29.6$ days for the sham group, with no significant differences between the groups (Table 1 ). 


\section{Pre- and post-treatment comparison}

The results from post-treatment CNT suggested that the leftFTAS group showed significant improvement in the backward digit span test, the verbal learning test-delayed recall, a visual span test, and the K-MMSE. The right-FTAS group showed improvement in verbal learning test-delayed recall. The sham group showed improvement in the backward visual span test. All three groups showed significant improvement in K-MBI scores (Table 2).

Table 1. Demographic characteristics of patients

\begin{tabular}{lccc}
\hline & Left-FTS & Right-FTAS & Sham \\
\hline Number of subject & 15 & 15 & 15 \\
Sex (male:female) & $6: 9$ & $7: 8$ & $7: 8$ \\
Age (year) & $60.9 \pm 12.9$ & $58.9 \pm 15.0$ & $68.5 \pm 14.6$ \\
Brain lesion & & & \\
Right & 6 & 6 & 8 \\
Left & 6 & 5 & 4 \\
Multiple & 3 & 4 & 3 \\
Cortical:subcortical & $6: 9$ & $8: 7$ & $10: 5$ \\
Infarction:hemorrhage & $7: 8$ & $9: 6$ & $10: 5$ \\
Post stroke duration (day) & $42.2 \pm 31.9$ & $38.1 \pm 27.0$ & $39.5 \pm 29.6$ \\
Education level & & & \\
Elementary school & 7 & 3 & 4 \\
Middle school & 0 & 3 & 1 \\
High school & 5 & 8 & 6 \\
University & 3 & 0 & 2 \\
Graduated school & 0 & 1 & 2 \\
\hline
\end{tabular}

Values: mean \pm standard deviation or number.

${ }^{*} P<0.05$ by Kruskal-Wallis test.

Left-FTAS, left fronto-temporal anode stimulation group; Right-FTAS, right frontotemporal anode stimulation group.

\section{Comparison between the three groups}

Comparison of pre- and post-treatment data for each group suggests that the left-FTAS group showed significant improvement in auditory memory, compared to the other groups. However, there were no significant differences between the groups in terms of visual memory and attention. There were no differences between the groups in terms of K-BMI (Table 3).

\section{Discussion}

The objective of this study was to determine the effect of tDCS treatment on the temporal lobe in terms of cognitive re-

Table 3. Comparison of improvement after treatment in each group

\begin{tabular}{lccc}
\hline & Left-FTAS & Right-FTAS & Sham \\
\hline$\Delta$ K-MMSE & $4.0 \pm 3.3$ & $2.3 \pm 5.3$ & $2.7 \pm 4.6$ \\
$\Delta$ FDST & $0.4 \pm 1.0$ & $0.4 \pm 1.5$ & $0.5 \pm 1.0$ \\
$\Delta$ BDST & $0.2 \pm 0.4$ & $0.1 \pm 0.8$ & $0.0 \pm 0.6$ \\
$\Delta$ FVST & $0.2 \pm 0.6$ & $0.0 \pm 1.4$ & $0.5 \pm 0.9$ \\
$\Delta$ BVST & $0.2 \pm 0.6$ & $0.3 \pm 1.2$ & $0.8 \pm 1.2$ \\
$\Delta$ ViLT $-R$ & $4.1 \pm 8.1$ & $3.0 \pm 9.6$ & $1.7 \pm 14.4$ \\
$\Delta$ VeLT-R & $10.5 \pm 6.1^{*}$ & $5.1 \pm 5.2$ & $5.4 \pm 9.8$ \\
$\Delta$ VCPT & $-0.02 \pm 0.2$ & $-0.02 \pm 0.2$ & $-0.01 \pm 0.1$ \\
$\Delta$ ACPT & $-0.03 \pm 0.2$ & $-0.01 \pm 0.2$ & $-0.02 \pm 0.1$ \\
$\Delta$ K-MBI & $9.5 \pm 7.5$ & $10.5 \pm 10.5$ & $7.5 \pm 6.8$
\end{tabular}

Values: mean \pm standard deviation

${ }^{*} P<0.05$ by Kruskal-Wallis test.

Left-FTAS, left frontotemporal anode stimulation group; Right-FTAS, right frontotemporal anode stimulation group; K-MMSE, Korean version of the Mini-mental status examination; FDST, forward digit span test; BDST, backward digit span test; FVST, forward visual span test; BVST, backward visual span test; ViLT-R, visual learning test-delayed recall; VeLT-R, verbal learning test-delayed recall; VCPT, visual continuous performance test; ACPT, auditory continuous performance test; $\mathrm{K}-\mathrm{MBI}$, Korean version of the modified Bathel Index.

Table 2. Changes in cognitive function after treatment

\begin{tabular}{|c|c|c|c|c|c|c|}
\hline & \multicolumn{2}{|c|}{ Left-FTAS } & \multicolumn{2}{|c|}{ Right-FTAS } & \multicolumn{2}{|c|}{ Sham } \\
\hline & Before & After & Before & After & Before & After \\
\hline K-MMSE & $20.1 \pm 4.8$ & $23.9 \pm 3.0^{*}$ & $20.8 \pm 5.6$ & $23.1 \pm 3.2$ & $19.0 \pm 5.2$ & $21.7 \pm 4.1^{*}$ \\
\hline FDST & $4.6 \pm 1.4$ & $5.0 \pm 1.2$ & $4.4 \pm 1.4$ & $4.8 \pm 1.7$ & $4.4 \pm 1.2$ & $4.9 \pm 1.4$ \\
\hline BDST & $2.8 \pm 0.9$ & $3.0 \pm 0.9^{*}$ & $3.1 \pm 1.2$ & $3.3 \pm 1.4$ & $2.9 \pm 1.0$ & $2.9 \pm 1.0$ \\
\hline FVST & $3.3 \pm 0.5$ & $3.6 \pm 0.8^{*}$ & $3.7 \pm 1.2$ & $3.8 \pm 0.8$ & $3.3 \pm 0.5$ & $3.8 \pm 1.0$ \\
\hline BVST & $2.6 \pm 1.2$ & $3.3 \pm 1.4$ & $2.7 \pm 1.0$ & $3.0 \pm 1.2$ & $2.3 \pm 0.4$ & $3.0 \pm 1.5^{*}$ \\
\hline VilT-R & $32.6 \pm 17.0$ & $36.7 \pm 15.7$ & $31.0 \pm 17.5$ & $34.0 \pm 17.0$ & $31.1 \pm 17.5$ & $32.2 \pm 15.4$ \\
\hline VeLT-R & $16.1 \pm 11.2$ & $27.6 \pm 14.3^{*}$ & $18.4 \pm 10.4$ & $24.1 \pm 8.7^{*}$ & $20.6 \pm 9.0$ & $26.8 \pm 15.5$ \\
\hline VCPT (second) & $0.5 \pm 0.1$ & $0.5 \pm 0.1$ & $0.5 \pm 0.2$ & $0.5 \pm 0.1$ & $0.6 \pm 0.1$ & $0.6 \pm 0.2$ \\
\hline ACPT (second) & $0.7 \pm 0.1$ & $0.6 \pm 0.2$ & $0.6 \pm 0.2$ & $0.6 \pm 0.1$ & $0.7 \pm 0.1$ & $0.7 \pm 0.1$ \\
\hline K-MBI & $59.0 \pm 22.7$ & $68.7 \pm 21.2^{*}$ & $64.4 \pm 24.7$ & $74.9 \pm 22.5^{*}$ & $56.8 \pm 24.7$ & $64.4 \pm 23.2^{*}$ \\
\hline
\end{tabular}

Values: mean \pm standard deviation.

${ }^{*} P<0.05$ by Wilcoxon signed-rank test.

Left-FTAS, left fronto-temporal anode stimulation group; Right-FTAS, right fronto-temporal anode stimulation group; K-MMSE, Korean version of the Mini-Mental State Examination; FDST, forward digit span test; BDST, backward digit span test; FVST, forward visual span test; BVST, backward visual span test; ViLT-R, visual learning test-delayed recall; VeLT-R, verbal learning test-delayed recall; VCPT, visual continuous performance test; ACPT, auditory continuous performance test; K-MBI, Korean version of the modified Barthel Index. 
covery of poststroke patients. To date, only a small number of studies have been done on $\mathrm{tDCS}$ as a treatment method for cognitive impairment. While Montie et al. suggested that cathode stimulation to the left fronto-temporal lobe resulted in improvement in naming in aphasia, ${ }^{12}$ other studies concluded that anode stimulation to the left perisylvian region resulted improvement in word memory in healthy patients. ${ }^{13,14}$ Boggio et al. also concluded that anode stimulation to the left fronto-temporal lobe helped to improve memory performance in Alzheimer disease. ${ }^{10,11}$ The results of our study suggested that anodal stimulation to the left fronto-temporal lobe improved auditory memory, confirming the conclusion of earlier studies, although the treatment did not affect visual memory. CNT test showed that the treatment had been positively effective in all areas, but other three groups, excluding auditory memory, showed no statistical difference.

Only few studies have been done on the effect of tDCS on cognitive function in stroke patients. While Kang et al. ${ }^{8}$ suggested that anode stimulation at the dorso-lateral prefrontal cortex improved attention, Kim et al. ${ }^{15}$ concluded that rTMS on the same region was only effective in treating depression, and did not affect cognitive function. There have been two reports on patients with stroke who experienced linguistic improvement, ${ }^{15}$ but these studies only involved 10 and 13 patients, respectively. No studies have clearly defined the effects of noninvasive brain stimulation on the cognitive function of stroke patients.

Studies have discovered that $\mathrm{tDCS}$ affects the brain by activating N-methyl-D-aspartic acid (NMDA) receptors and suppressing the cerebral cortex via gamma-aminobutyric acid (GABA) receptors. ${ }^{16,17}$ The effect of $\mathrm{tDCS}$ is known to last for approximately 90 minutes, during which the cerebral cortex remains excited, ${ }^{18}$ but there are too few studies to be able to draw conclusions on the accumulated effect. This report showed that marked improvements are noted after 3 weeks of $\mathrm{tDCS}$ treatment, but the accumulated effect could not be determined because serial cognitive function was not assessed. Future studies should monitor cognitive function serially to observe whether the improvements are gradual.

The findings from this study may have been limited by the small number of patients in each test group. Cerebral infarction and hemorrhage were both included and lesions varied largely, so that spontaneous recovery could not be fully excluded. As well as excluding cathode stimulation from the treatment. Additionally, the subjects were not tested for depression, which may have affected the outcome. As it has been suggested that depression is prominent among stroke patients and as depression may affect attention and memory, ${ }^{19}$ the possibility remains that the study may have included patients with depression, whose de- pression symptoms were affected by $\mathrm{tDCS}$, which then subsequently resulted in improved cognitive function. Furthermore, poststroke apathy can affect cognitive function, but this could not be addressed in this study. Since the subjects were evaluated for cognitive performance at 24 hours after completion of the 3-week treatment program, the long-term effect of tDCS treatment remains undetermined. Moreover, we excluded patients with abnormal cognitive function based on the guardian's statement, but we had not tested the cognitive function of patients before they suffered their stroke, which may have an impact on the study result.

Nevertheless, this study confirmed that tDCS is effective in treating poststroke cognitive impairment. We were also able to conclude that stimulation on the temporal lobe, as well as on the prefrontal lobe, as suggested by earlier studies, is helpful in cognitive recovery.

\section{Conclusions}

The results from this study confirmed that applying tDCS to the left temporal lobe effectively improved auditory memory of patients with poststroke cognitive impairment. We concluded that $\mathrm{tDCS}$ can be employed as a treatment sub-protocol in a cognitive treatment program. However, further studies with a larger subject pool and various stimulation methods should be conducted to confirm these findings.

\section{References}

1. Diamond PT, Felsenthal G, Macciocchi SN, Butler DH, LallyCassady D. Effect of cognitive impairment on rehabilitation outcome. Am J Phys Med Rehabil 1996;75:40-43.

2. Pohjasvaara T, Erkinjuntti T, Vataja R, Kaste M. Dementia three months after stroke. Baseline frequency and effect of different definitions of dementia in the Helsinki Stroke Aging Memory Study (SAM) cohort. Stroke 1997;28:785-792.

3. Song X, Mitnitski A, Rockwood K. Prevalence and 10-year outcomes of frailty in older adults in relation to deficit accumulation. J Am Geriatr Soc 2010;58:681-687.

4. Tatemichi TK, Desmond DW, Mayeux R, Paik M, Stern Y, Sano M, et al. Dementia after stroke: baseline frequency, risks, and clinical features in a hospitalized cohort. Neurology 1992; 42:1185-1193.

5. Kim YH, Jang EH, Lee SJ, Park JW, Ko MH, Park SH, et al. Development of computer-assisted memory rehabilitation programs for the treatment of memory dysfunction in patients with brain injury. J Korean Acad Rehab Med 2003;27:667-674.

6. Boggio PS, Nunes A, Rigonatti SP, Nitsche MA, Pascual-Le- 
one A, Fregni F. Repeated sessions of noninvasive brain DC stimulation is associated with motor function improvement in stroke patients. Restor Neurol Neurosci 2007;25:123-129.

7. Smith DV, Clithero JA. Manipulating executive function with transcranial direct current stimulation. Front Integr Neurosci 2009;3:26.

8. Kang EK, Baek MJ, Kim S, Paik NJ. Non-invasive cortical stimulation improves post-stroke attention decline. Restor Neurol Neurosci 2009;27:645-650.

9. Chi RP, Fregni F, Snyder AW. Visual memory improved by non-invasive brain stimulation. Brain Res 2010;1353:168-175.

10. Boggio PS, Khoury LP, Martins DC, Martins OE, de Macedo EC, Fregni F. Temporal cortex direct current stimulation enhances performance on a visual recognition memory task in Alzheimer disease. J Neurol Neurosurg Psychiatry 2009;80:444-447.

11. Boggio PS, Fregni F, Valasek C, Ellwood S, Chi R, Gallate J, et al. Temporal lobe cortical electrical stimulation during the encoding and retrieval phase reduces false memories. PLoS One 2009;4:e4959.

12. Monti A, Cogiamanian F, Marceglia S, Ferrucci R, Mameli F, Mrakic-Sposta $S$, et al. Improved naming after transcranial direct current stimulation in aphasia. J Neurol Neurosurg Psychiatry 2008;79:451-453.
13. Floel A, Rosser N, Michka O, Knecht S, Breitenstein C. Noninvasive brain stimulation improves language learning. J Cogn Neurosci 2008;20:1415-1422.

14. Sparing R, Mottaghy FM. Noninvasive brain stimulation with transcranial magnetic or direct current stimulation (TMS/ tDCS)_From insights into human memory to therapy of its dysfunction. Methods 2008;44:329-337.

15. Kim BR, Kim DY, Chun MH, Yi JH, Kwon JS. Effect of repetitive transcranial magnetic stimulation on cognition and mood in stroke patients: a double-blind, sham-controlled trial. Am J Phys Med Rehabil 2010;89:362-368.

16. Liebetanz D, Nitsche MA, Tergau F, Paulus W. Pharmacological approach to the mechanisms of transcranial DC-stimulation-induced after-effects of human motor cortex excitability. Brain 2002;125:2238-2247.

17. Chen R. Interactions between inhibitory and excitatory circuits in the human motor cortex. Exp Brain Res 2004;154:1-10.

18. Nitsche MA, Paulus W. Sustained excitability elevations induced by transcranial DC motor cortex stimulation in humans. Neurology 2001;57:1899-1901.

19. Tateno A, Kimura M, Robinson RG. Phenomenological characteristics of poststroke depression: early- versus late-onset. Am J Geriatr Psychiatry 2002;10:575-582. 